\title{
Microbial Profiles and Detection Techniques in Peri-Implant Diseases: a Systematic Review
}

\author{
Miguel Padial-Molina ${ }^{1}$, Jesús López-Martínez ${ }^{1}$, Francisco O’'Valle ${ }^{2}$, Pablo Galindo-Moreno ${ }^{1}$ \\ ${ }^{1}$ Department of Oral Surgery and Implant Dentistry, School of Dentistry, University of Granada, Spain. \\ ${ }^{2}$ Department of Pathology and Biopathology and Medicine Regenerative Institute (IBIMER, CIBM), University of Granada, \\ Spain.
}

\author{
Corresponding Author: \\ Pablo Galindo-Moreno \\ C/ Recogidas, $395^{\circ}$ Izq. - 18005 Granada \\ Spain \\ Phone: +34 958520658 \\ E-mail: pgalindo@ugr.es
}

\begin{abstract}
Objectives: To describe the microbial profiles of peri-implant diseases and the main detection methods.

Material and Methods: A literature search was performed in MEDLINE via PubMed database to identify studies on microbial composition of peri-implant surfaces in humans published in the last 5 years. Studies had to have clear implant status definition for health, peri-implant mucositis and/or peri-implantitis and specifically study microbial composition of the peri-implant sulcus.

Results: A total of 194 studies were screened and 47 included. Peri-implant sites are reported to be different microbial ecosystems compared to periodontal sites. However, differences between periodontal and peri-implant health and disease are not consistent across all studies, possibly due to the bias introduced by the microbial detection technique. New methods non species-oriented are being used to find 'unexpected' microbiota not previously described in these scenarios.

Conclusions: Microbial profile of peri-implant diseases usually includes classic periodontopathogens. However, correlation between studies is difficult, particularly because of the use of different detection methods. New metagenomic techniques should be promoted for future studies to avoid detection bias.
\end{abstract}

Keywords: dental implants; diagnosis; microbiology; peri-implantitis.

\author{
Accepted for publication: 7 September 2016 \\ To cite this article: \\ Padial-Molina M, López-Martínez J, O’Valle F, Galindo-Moreno P. \\ Microbial Profiles and Detection Techniques in Peri-Implant Diseases: a Systematic Review \\ J Oral Maxillofac Res 2016;7(3):e10 \\ URL: http://www.ejomr.org/JOMR/archives/2016/3/e10/v7n3e10.pdf \\ doi: $10.5037 /$ jomr.2016.7310
}




\section{INTRODUCTION}

Peri-implant diseases are not an emerging group of diseases. Peri-implant diseases are a prevalent reality: 19 to $65 \%$ of implants present peri-implant mucositis (weighted mean of $43 \%$ [CI $=32$ to $54 \%]$ ) and 1 to $47 \%$ develop peri-implantitis (weighted mean of $22 \%$ [CI $=14$ to $30 \%]$ ) alongside a positive relationship with function time [1]. Moreover, periimplant diseases are challenging to treat [2] , and, in some cases, with aggressive progression patterns [3]. However, consensus has been reached so far only on few facts associated with increased risk of peri-implant disease development: 1) lack of regular supportive therapy; 2) plaque accumulation; 3) smoking; 4) history of periodontal disease; and 5) excess cement [4]. Fortunately, it has been established that patient-administered mechanical plaque control (with manual or powered toothbrushes) and professional intervention comprising oral hygiene instructions and mechanical debridement are adequate measures to reduce peri-implant mucositis and its progression to peri-implantitis [ㅁ]. Several strategies are being investigated to reduce plaque accumulation over implants: surface modifications [6-11], lasers and other physical methods [12-16], locally delivered antibiotics [17-19], and even better seals of the connection implant-abutment to reduce this particular reservoir of microbiota [20-23]. However, these and other adjunctive measures to reduce plaque, including systemic antibiotics, have not been found to reduce clinical signs of inflammation $[\underline{5}, \underline{24}]$.

So far, the main diagnostic methods of peri-implant disease are based on clinical and radiographic data. These data are obviously insufficient and they only detect the disease when it has produced some level of destruction. Thus, early diagnosis and identification of risk factors are of extreme importance to prevent the disease in the first place. Probably the more studied risk factor for peri-implant disease is the microbiota associated with the peri-implant sulcus. In fact, the presence of periodontophatic bacteria in the periimplant sulcus has been proposed as a risk indicator for both peri-implant mucositis and peri-implantitis $[\underline{4}, \underline{25}]$. The characteristics of the sulcus environment favour its colonization by anaerobic Gram-negative bacteria. The ecological succession of the microbes in the sulcus may lead to the development of the disease. The peri-implant disease has been described as poly-microbial anaerobic infection similar to that found in chronic periodontitis $[\underline{3}, \underline{26}, \underline{27}]$. However, new technologies are finding an increasing number of microorganisms in peri-implant sites not found around teeth [4].

Many studies have evaluated the microbiota around healthy and diseased teeth as well as around healthy and diseased implants by different techniques. Thus, this systematic narrative review is aimed at identifying the microbiological factors that have been associated with the presence of peri-implant disease by describing the findings published and discussing the main detection techniques.

\section{MATERIAL AND METHODS Protocol and registration}

The methods of the analysis and inclusion criteria were specified in advance and documented in a protocol. The review was registered in PROSPERO, Registration number: CRD42016037647.

The reporting of this systematic analysis adhered to the Preferred Reporting Items for Systematic Review and Meta-Analyses (PRISMA) statement [28].

\section{Focus question}

The following focus question was developed according to the population, intervention, comparison, and outcome (PICO) study design: what are the microbial profiles of human patients suffering periimplantitis in comparison to healthy implants? Additionally, the comparisons with implants suffering mucositis and with healthy or diseased teeth were also explored.

\section{Information sources}

A literature search was performed in MEDLINE via PubMed database of the US National Library of Medicine on April 25 $5^{\text {th }}, 2016$ using Medical Subject Heading search terms and free text terms in different combinations. A hand search was also performed in dental and implant-related journals from April 2011 to April 2016, including "Journal of Dental Research", "Journal of Clinical Periodontology", "Clinical Oral Implants Research", "Journal of Periodontology", "International Journal of Oral and Maxillofacial Implants", "Journal of Oral Implantology", "Implant Dentistry", "Clinical Implant Dentistry and Related Research", "European Journal of Oral Implantology", "International Journal of Oral and Maxillofacial Surgery", "Journal of Oral and Maxillofacial Surgery", "Journal of Prosthetic Dentistry", "International Journal of Prosthodontics", "International Journal of Periodontics \& Restorative Dentistry". 
The search was limited to English language articles. A hand search of the reference lists in the articles retrieved was also carried out for additional relevant publications.

\section{Search}

The electronic search strategy and limits was: ((("Microbiology") OR ("Microbiome") OR ("Microbial")) AND ("Dental Implants" [Mesh])) AND (("English and humans" [Filter]) AND ("published last 5 years" [Filter])) NOT ("review" [Filter]).

\section{Selection of studies}

The articles, at any stage (abstract or full-text assessment) were independently reviewed by 2 of the authors. Reviewers compared decisions and resolved differences through discussion and consulting the other authors when consensus could not be reached.

\section{Types of publications}

Studies on humans published in the English language were selected. Letters, editorials, case reports, literature reviews, and $\mathrm{PhD}$ theses were excluded.

\section{Types of studies}

No limitations as to the type of study design were established.

\section{Types of participants/population}

Included studies describe findings from human participants with at least one osseointegrated titanium screw-shaped dental implant with signs of periimplantitis or peri-implant mucositis, with or without healthy implants or teeth.

\section{Disease definition}

Peri-implant mucositis or peri-implantitis must have been defined in each article according to the current classification of peri-implant diseases [29].

\section{Inclusion and exclusion criteria}

To be included in the study, studies had to have clear implant status definition for the conditions health, mucositis and/or peri-implantitis and analyse the microbiome of those situations, with or without comparisons among them or with or without before and after results.

The applied exclusion criteria for studies were animal or in vitro studies, not enough information on the microbial analysis, analysis not performed on periimplant sulcus of dental implants aimed at supporting restorations and no access to the abstract or full-text.

\section{Sequential search strategy}

Following the initial literature search, all article titles were screened to eliminate irrelevant publications, review articles, case reports, in vitro, and animal studies. Next, studies were excluded based on data obtained from screening the abstracts. The final stage of screening involved reading the full texts to confirm each study's eligibility, based on the inclusion and exclusion criteria.

\section{Data extraction}

The data was independently extracted from studies by two authors. Data extracted was descriptive findings of microbial profiles around dental implants and additional information such as findings around teeth with or without disease.

\section{Data items}

Data collected from the included articles were:

- Full reference - identification of study;

- Type of patients/sites - healthy implants, periimplant mucositis or peri-implantitis, and healthy or diseased teeth;

- Number of patients included in the study for each condition;

- Number of implants/sites included in the study for each condition;

- Outcome - description of the main findings of the study;

- Detection method - how the microbial profile was evaluated (culture, polymerase chain reaction (PCR), checkerboard or metagenomics).

\section{Risk of bias assessment}

Risk of bias within articles was assessed independently, and in duplicate by the authors according to the RTI Item Bank guide for bias and confounding assessment in observational studies of interventions or exposures [30].

Possible sources of bias include: inclusion/ exclusion criteria, recruitment of participants, selection of the comparison group, variations in the execution of the study from the proposed protocol, 
blindness to the outcome, exposure, or intervention, valid and reliable measures, length of follow-up, impact of missing data, missing primary outcomes, harms or adverse events, balance between the groups or match groups, and confounding variables.

Table 1 summarizes the findings for each of the included articles. An overall risk of bias was finally assigned to each report.

\section{Statistical analysis}

No meta-analysis was intended as no common microbial detection methodology is used across studies.

\section{RESULTS}

\section{Study selection}

Figure 1 shows the PRISMA flow diagram of studies identified, screened and included. The initial electronic and hand search retrieved 194 citations after duplicates were removed. These 194 were screened by titles and abstracts, from which 134 were excluded. Thus, 60 articles were assessed in full text. Of these, 14 were excluded: no analysis of peri-implant sulcus $=4$ [31-34]; not performed in oral mucosa $=2[\underline{35}]$; no full-text available $=1[\underline{36}]$; not enough microbiological data $=3$ [37-39]; not in conventional dental implants $=4$ [40-44]. Finally, 46 studies were included in the qualitative synthesis and analysis, as described in Tables 1 - 5 .

\section{Study characteristics}

Studies on peri-implant microbiota using culture techniques are limited in the last 5 years (Table 1).
With no doubt, this clearly reflects the incorporation of new technologies in the peri-implant diagnosis field as their used is declining. Only 4 studies have been identified using this methodology. It is concluded that Streptococcus spp. and Peptostreptococcus spp. are correlated with the increase in BOP 1 to 6 months after loading [45]. Similarly, there is a significantly higher prevalence of Porphyromonas, Prevotella and anaerobic Gram positive cocci in peri-implantitis vs. peri-implant health [46]. In contrast, a reduction on spirochetes was found from implant placement on periodontal sites to 12 months [47]. Using bacteria culture, similar effectiveness has been described when using placebo or chlorhexidine for the treatment of peri-implantitis sites [48].

A total of 14 studies have been identified using PCR techniques (Table 2). These studies, overall, fail to demonstrate similar patterns in terms of detected species, frequencies of detection as well as bacterial load. As an example, some studies found no differences in the detection frequencies of Aggregatibacter actinomycetemcomitans (A.a.), Porphyromonas gingivalis, Prevotella intermedia and Treponema denticola between healthy and diseased implants $[49,50]$. Others did find differences for A.a., P. gingivalis, Tannerella forsythia, T. denticola [51], Fusobacterium nucleatum, $P$. intermedia, Peptostreptococcus micros, Campylobacter rectus, Eikenella corrodens, Candida albicans, Prevotella nigrescens, Centruroides gracilis, Capnocytophaga ochracea, Campylobacter concisus, Streptococcus spp., Actinomyces odontolyticus, Veillonella parvula and Enterococcus faecalis [ [22, $\underline{53}$ ].

Compared to teeth, implants are usually reported to have less bacterial species; periodontitis is also reported to be more diverse than peri-implantitis [54]. Other studies did not find these differences $[50,55]$.

Table 1. Studies using culture techniques

\begin{tabular}{|c|c|c|c|c|c|}
\hline Study & $\begin{array}{c}\text { Year of } \\
\text { publication }\end{array}$ & $\begin{array}{c}\text { Type of } \\
\text { patients/sites }\end{array}$ & $\begin{array}{c}\text { Number of } \\
\text { patients }\end{array}$ & $\begin{array}{c}\text { Number of } \\
\text { implants/sites }\end{array}$ & Outcome \\
\hline $\begin{array}{l}\text { Asadzadeh et } \\
\text { al. [45] }\end{array}$ & 2012 & $\begin{array}{l}\text { Peri-implant } \\
\text { tissues }\end{array}$ & 20 patients & 20 implants & $\begin{array}{l}\text { Correlation between the increase in peri-implant } \\
\text { BOP from } 1 \text { to } 6 \text { months after loading and an } \\
\text { increase in colonies of Streptococcus spp. and Pep- } \\
\text { tostreptococcus spp. }\end{array}$ \\
\hline $\begin{array}{l}\text { Neilands et al. } \\
{[46]}\end{array}$ & 2015 & \begin{tabular}{|c|}
$\begin{array}{c}\text { Peri-implantitis } \\
\text { vs. peri-implant } \\
\text { health }\end{array}$ \\
\end{tabular} & \begin{tabular}{|c|}
50 patients \\
$(25$ peri-implantitis; \\
25 healthy $)$
\end{tabular} & $\begin{array}{l}1 \text { implant } \\
\text { per patient }\end{array}$ & $\begin{array}{l}\text { Highly variable microbial composition. Significantly } \\
\text { higher prevalence of Porphyromonas/Prevotella and } \\
\text { anaerobic Gram positive cocci in peri-implantitis. }\end{array}$ \\
\hline $\begin{array}{l}\text { Tripodakis et } \\
\text { al. [47] }\end{array}$ & 2011 & $\begin{array}{c}\text { Peri-implant } \\
\text { tissue vs. peri- } \\
\text { odontal disease }\end{array}$ & 20 patients & $\begin{array}{c}20 \text { periodontal sites } \\
\text { that became } \\
20 \text { peri-implant sites }\end{array}$ & $\begin{array}{l}\text { Spirochetes in peri-implant samples: } 69 \% \text { vs. } 2 \% \text { at } \\
\text { baseline and } 12 \text { months, respectively. Higher colony } \\
\text { forming units (CFU)/ml in samples from periodontal } \\
\text { sites. }\end{array}$ \\
\hline $\begin{array}{l}\text { de Waal et al. } \\
\text { [48] }\end{array}$ & 2013 & Peri-implantitis & $\begin{array}{c}30 \text { patients } \\
(15 \text { placebo; } 15 \text { test } \\
\text { [chlorhexidine] })\end{array}$ & $\begin{array}{c}79 \text { implants } \\
(48 \text { placebo; } 31 \text { test })\end{array}$ & $\begin{array}{l}\text { Sixty of the } 79 \text { implants were positive at baseline. } \\
\text { A.a. not detected. Both procedures reduced } P \text {. gin- } \\
\text { givalis, P. intermedia, T. forsythia, F. nucleatum, P. } \\
\text { micra and C. rectus. }\end{array}$ \\
\hline
\end{tabular}




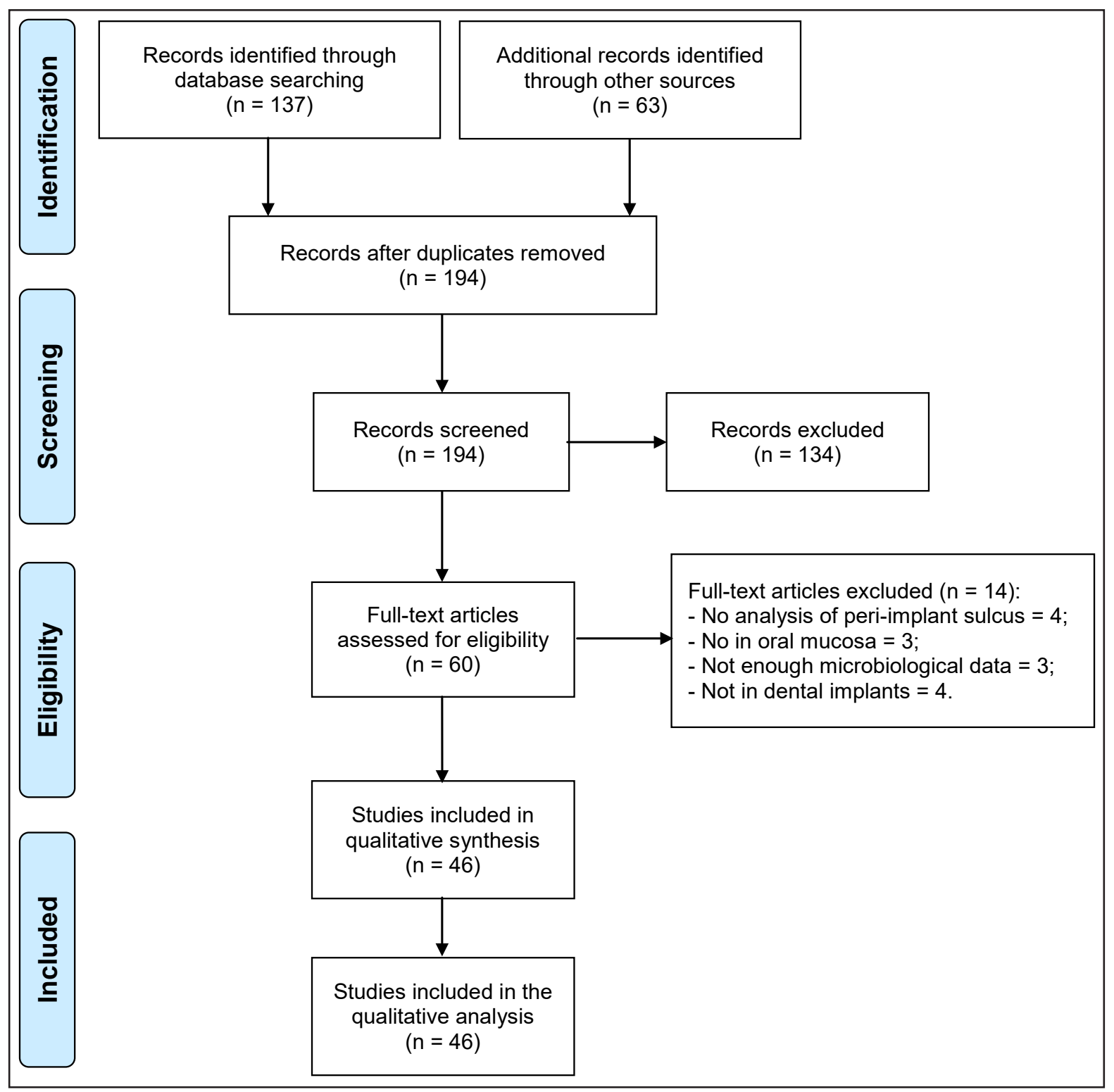

Figure 1. PRISMA flow diagram of included studies.

In any case, it seems that the presence of periodontal disease and implants increase the presence of periodontophatic bacteria in the peri-implant sulcus, which does not necessarily mean that these bacteria are involved in peri-implant disease [56]. Interestingly, Sato et al. found a higher number and detection rate of periodontopathic bacteria as the cumulative interceptive supportive therapy (CIST) level increased [57].

The next technique identified was checkerboard DNADNA. Eight studies have been published using this methodology (Table 3). Several differences have been found with this methodology. As expected, plaque and bleeding on probing scores have been significantly correlated with sulcular levels of bacteria [58], although the total DNA count was not significantly different between implants with shallow and deep pockets [59]. However, other studies involving a greater number of patients also found total DNA count to be correlated to interproximal bleeding index $(r=$ $0.409)$ and interproximal probing depth $(r=0.307)$ but no correlations were present with plaque index or radiographic bone level up to 22 years after implant placement $[\underline{60}]$. When comparing healthy vs. diseased implant sites, only $37.5 \%$ of all species showed a higher prevalence, although only 10 species were explored [61]. In contrast, only 14 species out of the 79 studied (17.7\%) were more prevalent in periimplantitis than in healthy sites in a study by Persson and Renvert [62] involving 166 peri-implantitis sites and 47 healthy implants. Interestingly, this study also found a cluster of T.forsythia, P.gingivalis, Treponemasocranskii, Staphylococcus aureus, Staphylococcus anaerobius, Staphylococcus intermedius, and Streptococcusmitis that comprised $30 \%$ of the total microbiota at peri-implantitis sites. 
Table 2. Studies using PCR techniques

\begin{tabular}{|c|c|c|c|c|c|}
\hline Study & $\begin{array}{c}\text { Year of } \\
\text { publication }\end{array}$ & $\begin{array}{c}\text { Type of } \\
\text { patients/sites }\end{array}$ & $\begin{array}{c}\text { Number of } \\
\text { patients }\end{array}$ & $\begin{array}{c}\text { Number of } \\
\text { implants/sites }\end{array}$ & Outcome \\
\hline Casado et al. [49] & 2011 & $\begin{array}{l}\text { Peri-implant health } \\
\text { vs. mucositis vs. peri- } \\
\text { implantitis }\end{array}$ & 30 subjects & $\begin{array}{c}30 \text { implants ( } 10 \text { healthy; } \\
10 \text { peri-implant mucositis; } \\
10 \text { peri-implantitis })\end{array}$ & No differences in A.a., P. gingivalis, P. intermedia, and T. denticola. \\
\hline Canullo et al. [50] & 2016 & $\begin{array}{l}\text { Peri-implant health vs. } \\
\text { peri-implantitis vs. } \\
\text { neighboring teeth }\end{array}$ & $\begin{array}{c}534 \text { patients } \\
\text { (53 peri-implantitis; } \\
481 \text { non-peri-implantitis) }\end{array}$ & $\begin{array}{c}1507 \text { implants } \\
\text { (231 peri-implantitis; } \\
1276 \text { non-peri-implantitis) }\end{array}$ & $\begin{array}{l}\text { No relevant differences between the healthy and disease implants in the same patient. } \\
\text { Similar in the neighboring teeth with a lower } P \text {. gingivalis, T. forsythia, P. intermedia, } P \text {. } \\
\text { micros, and E. corrodens. }\end{array}$ \\
\hline Wang et al. [51] & 2015 & $\begin{array}{l}\text { Peri-implant health vs. } \\
\text { peri-implantitis }\end{array}$ & $\begin{array}{c}68 \text { patients } \\
\text { (34 healthy; } 34 \text { peri-implantitis) }\end{array}$ & 1 per patient & $\begin{array}{l}\text { A.a., P. intermedia, P. gingivalis, T. forsythia and T. denticola associated with peri- } \\
\text { implantitis although no statistically significant. }\end{array}$ \\
\hline Canullo et al. [52] & 2015 & $\begin{array}{l}\text { Peri-implant health vs. } \\
\text { peri-implantitis vs. } \\
\text { neighboring teeth }\end{array}$ & $\begin{array}{c}110 \text { patients } \\
\text { (53 peri-implantitis; } \\
57 \text { non-peri-implantitis) }\end{array}$ & \begin{tabular}{|c|}
235 implants \\
(113 peri-implantitis; \\
122 non-peri-implantitis) \\
\end{tabular} & $\begin{array}{l}>20 \% \text { differences between healthy and disease implants for } T \text {. denticola and } E \text {. corrodens. } \\
\text { Marked differences between health and peri-implantitis for the red complex bacteria and } P \text {. } \\
\text { intermedia. }\end{array}$ \\
\hline Canullo et al. [53] & 2016 & $\begin{array}{l}\text { Peri-implant health vs. } \\
\text { peri-implantitis }\end{array}$ & $\begin{array}{c}47 \text { patients } \\
\text { (25 peri-implantitis; } \\
22 \text { non-peri-implantitis) }\end{array}$ & $\begin{array}{c}90 \text { implants } \\
(113 \text { peri-implantitis; } \\
122 \text { non-peri-implantitis })\end{array}$ & $\begin{array}{l}\text { Significantly higher } T \text {. forsythia, } T \text {. denticola, } F \text {. nucleatum, } P \text {. intermedia, } P . \text { micros, } C \text {. } \\
\text { rectus, E. corrodens, C. albicans, } P \text {. nigrescens, } C \text {. gracilis, } C \text {. ochracea, C. concisus, } \\
\text { Streptococcus spp., A. odontolyticus, V. parvula, and E. faecalis in peri-implantitis. }\end{array}$ \\
\hline Zhuang et al. [54] & 2016 & $\begin{array}{c}\begin{array}{c}\text { Peri-implant health and } \\
\text { disease vs. periodontal } \\
\text { health and disease }\end{array} \\
\end{array}$ & $\begin{array}{c}22 \text { patients with at least } 1 \text { diseased } \\
\text { implant, } 1 \text { diseased tooth, } 1 \text { healthy } \\
\text { implant and } 1 \text { healthy tooth }\end{array}$ & 1 per patient and condition & $\begin{array}{l}\text { S. aureus and F. nucleatum were the most commonly detected species. Only F. nucleatum } \\
\text { was more abundant in periodontitis. Only P. gingivalis and F. nucleatum were more } \\
\text { prevalent in periodontitis than peri-implantitis. }\end{array}$ \\
\hline Aoki et al. [55] & 2012 & $\begin{array}{c}\text { Implants vs. adjacent, } \\
\text { occluding and contralateral } \\
\text { teeth }\end{array}$ & 21 patients & NS & $\begin{array}{l}\text { No significant differences in A.a., P. intermedia, P. gingivalis, T. forsythia, T. denticola or } \\
\text { F. nucleatum. }\end{array}$ \\
\hline Cosgarea et al. [56] & 2012 & \begin{tabular}{|c|} 
Implants in chronic \\
periodontitis patients vs. \\
healthy
\end{tabular} & $\begin{array}{c}24 \text { patients } \\
\text { (11 periodontitis; } \\
13 \text { no periodontitis) }\end{array}$ & NS & $\begin{array}{l}\text { Higher P. gingivalis and A.a. at implants and teeth in the chronic periodontitis group. More } \\
\text { A.a., T. forsythia and F. nucleatum at teeth than at implants. PD and CAL correlated with } \\
\text { counts of P. gingivalis at teeth and implants. }\end{array}$ \\
\hline Sato et al. [57] & 2011 & Peri-implantitis vs. teeth & $\begin{array}{c}105 \text { patients with residual natural } \\
\text { teeth and implants with peri- } \\
\text { implantitis }\end{array}$ & 1 per patient and condition & $\begin{array}{l}\text { The number and detection rate of periodontopathic bacteria increased with CIST level. No } \\
\text { difference in P. gingivalis and T. denticola between CIST-B and CIST-C. Higher detection } \\
\text { rate of all periodontopathic bacteria for CIST-D. }\end{array}$ \\
\hline Canullo et al. [82] & 2015 & Implant-diseased individuals & 38 patients & 180 sites & 3 sites showed presence of E. faecalis and 1 showed presence of Pseudomonas aeruginosa. \\
\hline Jankovic et al. [83] & 2011 & $\begin{array}{l}\text { Peri-implant health } \\
\text { vs. mucositis vs. peri- } \\
\text { implantitis }\end{array}$ & $\begin{array}{l}80 \text { patients } \\
(25 \text { healthy; } 25 \text { mucositis; } \\
30 \text { peri-implantitis })\end{array}$ & 1 implant per patient & $\begin{array}{l}\text { HCMV-2 detected in } 53.3 \% \text { and EBV-1 in } 46.6 \% \text { of the peri-implantitis sites. HCMV-2 } \\
\text { not detected in healthy sites and EBV-1 in only one healthy site. Statistically significant } \\
\text { correlation between the presence of HCMV-2 and EBV-1 genotypes and clinical } \\
\text { parameters of peri-implantitis. }\end{array}$ \\
\hline Swierkot et al. [84] & 2013 & Healthy implants & $\begin{array}{c}83 \text { patients } \\
\text { (42 with sonic toothbrush; } \\
41 \text { with manual toothbrush) }\end{array}$ & 1 per patient and condition & $\begin{array}{l}\text { No significant changes in the microbiological parameters. Both groups exhibited a small } \\
\text { increase in total bacterial load at implants and teeth. }\end{array}$ \\
\hline van Brakel et al. [85] & 2011 & $\begin{array}{l}\text { Peri-implant tissues at } \\
\text { zirconia and titanium } \\
\text { implants }\end{array}$ & 22 patients & $\begin{array}{l}1 \text { per patient and type of } \\
\text { abutment }\end{array}$ & $\begin{array}{l}\text { Similar A.a., P. gingivalis, P. intermedia, T. forsythia, P. micros, F. nucleatum and } T \text {. } \\
\text { denticola at } 2 \text { weeks and } 3 \text { months. }\end{array}$ \\
\hline
\end{tabular}

$\mathrm{NS}=$ not specified; CIST $=$ cumulative interceptive supportive therapy $; \mathrm{PD}=$ probing depth $\mathrm{CAL}=$ clinical attachment level $; \mathrm{HCMV}=$ human cytomegalovirus; $\mathrm{EBV}=\mathrm{Epstein}-\mathrm{Barr}$ virus. 
Table 3. Studies using checkerboard DNA-DNA hybridization

\begin{tabular}{|c|c|c|c|c|c|}
\hline Study & $\begin{array}{c}\text { Year of } \\
\text { publication }\end{array}$ & $\begin{array}{c}\text { Type of } \\
\text { patients/sites }\end{array}$ & $\begin{array}{l}\text { Number of } \\
\text { patients }\end{array}$ & $\begin{array}{l}\text { Number of } \\
\text { implants/sites }\end{array}$ & Outcome \\
\hline Cosyn et al. [58] & 2011 & Peri-implant sulcus & 8 healthy patients & NS & $\begin{array}{l}\text { Plaque and BOP correlated with sulcular levels of } 30 / 40 \text { species. For } 25 \\
\text { species, the detection frequency was at least } 75 \% \text {. }\end{array}$ \\
\hline De Bruyn et al. [59] & 2013 & $\begin{array}{l}\text { Peri-implant bone } \\
\text { level }\end{array}$ & 12 patients & 71 implants & $\begin{array}{l}25 / 40 \text { species in more than } 80 \% \text { of the implants. Large variation in bacterial } \\
\text { levels between implants. Fusobacteria, Leptotrichia buccalis, } P \text {. micra, } V \text {. } \\
\text { parvula, and } T \text {. forsythia showed the highest levels. Implants with shallow } \\
\text { pockets showed significantly lower counts of: } \text { A. odontolyticus, } C \text {. gracilis, } \\
\text { F. nucleatum naviforme, and Leptotrichia buccalis. No significantly different } \\
\text { total DNA count between implants with shallow and deep pockets. }\end{array}$ \\
\hline Dierens et al. [60] & 2013 & $\begin{array}{l}\text { Peri-implant vs. } \\
\text { periodontal health }\end{array}$ & 46 patients & NS & $\begin{array}{l}\text { T. forsythia and } V \text {. parvula showed the highest concentrations around implants } \\
\text { and teeth, respectively. Significantly more } P \text {. gingivalis, } P \text {. intermedia, } T \text {. } \\
\text { forsythia, } P \text {. micra and } T \text {. denticola around implants. Total DNA count } \\
\text { correlated to interproximal bleeding index }(\mathrm{r}=0.409) \text { and interproximal } \\
\text { probing depth }(\mathrm{r}=0.307) \text {. No correlations with plaque index or radiographic } \\
\text { bone level. }\end{array}$ \\
\hline Ebadian et al. [61] & 2012 & $\begin{array}{l}\text { Peri-implant health } \\
\text { and disease vs. } \\
\text { periodontal health and } \\
\quad \text { disease }\end{array}$ & $\begin{array}{l}\quad 69 \text { patients } \\
\text { (21 non-periodontitis; } \\
22 \text { chronic periodontitis; } \\
13 \text { non-peri-implantitis; } \\
13 \text { peri-implantitis) }\end{array}$ & 1 per patient & $\begin{array}{l}\text { Statistical difference between prevalence of } P \text {. intermedia, } P \text {. gingivalis, } T \text {. } \\
\text { forsythia, C. rectus, Prevotella tannerae, } T \text {. denticola and } P \text {. endonticula in all } \\
\text { groups. Higher incidence of all species in periodontitis sites; only } 37.5 \% \text { of } \\
\text { species showed higher prevalence in peri-implantitis. Significant difference for } \\
\text { T. forsythia, } P \text {. intermedia and } C \text {. rectus in PI vs. CP; no significant difference } \\
\text { between HI and HP. }\end{array}$ \\
\hline Persson et al. [62] & 2014 & $\begin{array}{l}\text { Peri-implantitis vs. } \\
\text { peri-implant health }\end{array}$ & $\begin{array}{l}213 \text { patients } \\
\text { (166 peri-implantitis; } \\
47 \text { healthy) }\end{array}$ & 1 implant per patient & $\begin{array}{l}\text { Higher counts of A.a., C. gracilis, C. rectus, Campylobacter showae, } \\
\text { Helicobacter pylori, Haemphilus influenzae, P. gingivalis, S. aureus, S. } \\
\text { anaerobius, S. intermedius, S. mitis, T. forsythia, T. denticola and T. socranskii } \\
\text { in peri-implantitis. A cluster including T. forsythia and } S . \text { aureus are associated } \\
\text { with peri-implantitis. }\end{array}$ \\
\hline Salvi et al. [63] & 2012 & $\begin{array}{l}\text { Peri-implant mucositis } \\
\text { vs. periodontal } \\
\text { gingivitis }\end{array}$ & 15 patients & $\begin{array}{l}1 \text { per patient and } \\
\text { condition }\end{array}$ & $\begin{array}{l}\text { No differences in total DNA counts or detection frequency for putative } \\
\text { periodontal pathogens between implant and tooth sites. P. gingivalis detected } \\
\text { occasionally after } 3 \text { weeks of abolished oral hygiene. }\end{array}$ \\
\hline Hallström et al. [86] & 2012 & Peri-implant mucositis & $\begin{array}{c}43 \text { patients ( } 21 \text { control; } \\
22 \text { test [systemic antibiotics]) }\end{array}$ & 1 per patient & No differences between groups. \\
\hline Tsoukaki et al. [87] & 2013 & $\begin{array}{l}\text { Flapped vs. flapless } \\
\text { implants }\end{array}$ & $\begin{array}{l}20 \text { patients } \\
\text { (10 flapped; } 10 \text { flapless) }\end{array}$ & $\begin{array}{l}30 \text { implants } \\
\text { (15 flapped; } 15 \text { flapless) }\end{array}$ & $\begin{array}{l}\text { Significantly higher levels of } P \text {. gingivalis and } T \text {. forsythia and higher but not } \\
\text { significant } T \text {. denticola in flapless vs. flapped implants. }\end{array}$ \\
\hline
\end{tabular}

$\mathrm{NS}=$ not specified; $\mathrm{BOP}=$ bleeding on probing; $\mathrm{PI}=$ peri-implantitis $\mathrm{CP}=$ chronic periodontitis $\mathrm{HI}=$ healthy implant $\mathrm{HP}=$ healthy periodontium. 
Table 4. Studies using $16 \mathrm{~S}$ rRNA gene sequencing techniques

\begin{tabular}{|c|c|c|c|c|c|}
\hline Study & $\begin{array}{c}\text { Year of } \\
\text { publication }\end{array}$ & $\begin{array}{c}\text { Type of } \\
\text { patients/sites }\end{array}$ & $\begin{array}{c}\text { Number of } \\
\text { patients }\end{array}$ & $\begin{array}{c}\text { Number of } \\
\text { implants/sites }\end{array}$ & Outcome \\
\hline Tamura et al. [64] & 2013 & $\begin{array}{l}\text { Peri-implant health vs. } \\
\text { disease }\end{array}$ & 30 patients (15 healthy; 15 peri-implantitis) & 1 per patient & $\begin{array}{l}\text { Healthy sites: Streptococcus, Pseudoramibacter alactolyticus, Actinomyces israelii, Propionibacterium acnes, and } P \text {. } \\
\text { micra. } \\
\text { Peri-implantitis: Eubacterium nodatum, Eubacterium brachy, Eubacterium saphenum, Filifactor alocis, Spodoptera } \\
\text { exigua, Parassardovia denticolens, } P \text {. intermedia, F nucleatum, } P \text {. gingivalis, Centipeda periodontii, and } P \text {. micra. } \\
\text { Peri-implantitis sites had approximately 10-fold higher CFU/ml than healthy sites. }\end{array}$ \\
\hline Cortelli et al. [65] & 2013 & $\begin{array}{l}\text { Peri-implant health and } \\
\text { disease vs. periodontal } \\
\text { health and disease }\end{array}$ & $\begin{array}{l}306 \text { patients ( } 53 \text { peri-implant health; } 50 \text { peri-implant } \\
\text { mucositis; } 50 \text { peri-implantitis; } 50 \text { gingivitis; } \\
\qquad 5 \text { periodontitis) }\end{array}$ & NS & $\begin{array}{l}\text { Higher bacterial frequency in peri-implantitis than health; similar frequencies in peri-implantitis and mucositis; } \\
\text { higher bacterial frequency at teeth. } \\
\text { P. gingivalis and red complex: more common in peri-implantitis than mucositis. } \\
\text { C. rectus and } T \text {. forsythia: more frequent in healthy teeth/gingivitis than healthy implants/mucositis. } \\
\text { P. gingivalis and A.a.: similar in periodontitis and peri-implantitis. } \\
\text { Other species: higher in periodontitis than peri-implantitis. }\end{array}$ \\
\hline Zheng et al. [66] & 2015 & $\begin{array}{l}\text { Peri-implant health vs. } \\
\text { disease }\end{array}$ & $\begin{array}{c}24 \text { patients (10 healthy; } 8 \text { peri-implant mucositis; } \\
6 \text { peri-implantitis) }\end{array}$ & 1 per patient & $\begin{array}{l}\text { Higher diversity in ailing implants, vs. healthy implants. } P \text {. gingivalis, T. forsythia and } P \text {. intermedia clustered in } \\
\text { peri-implant mucositis. Peri-implantitis pathogens were present in peri-implant mucositis. Microbiome of mucositis } \\
\text { were intermediate in nature between healthy and peri-implantitis. }\end{array}$ \\
\hline Tsigarida et al. [67] & 2015 & $\begin{array}{l}\text { Peri-implant health vs. } \\
\text { disease }\end{array}$ & $\begin{array}{l}80 \text { patients }(40 \text { peri-implant health }[20 \text { smokers and } \\
20 \text { non-smokers]; } 20 \text { peri-implant mucositis [10 smokers } \\
\text { and } 10 \text { non-smokers]; } 20 \text { peri-implantitis [10 smokers and } \\
10 \text { non-smokers }])\end{array}$ & 1 per patient and condition & $\begin{array}{l}\text { Lower diversity with higher disease-associated species in healthy sites of smokers. Shifts from health to mucositis } \\
\text { accompanied by loss of several health-associated species. Peri-implantitis did not differ from mucositis. In non- } \\
\text { smokers, the shift from health to mucositis increased diversity. Few differences were detected between peri- } \\
\text { implantitis and mucositis. }\end{array}$ \\
\hline Koyanagi et al. [68] & 2013 & $\begin{array}{l}\text { Peri-implant disease vs. } \\
\text { periodontal disease }\end{array}$ & 6 patients & 12 sites (6 implants; 6 teeth) & $\begin{array}{l}\text { More diverse microbial composition of peri-implantitis than periodontitis. } \\
\text { P. micra only in peri-implantitis. }\end{array}$ \\
\hline da Silva et al. [69] & 2014 & $\begin{array}{l}\text { Peri-implantitis vs. healthy } \\
\text { implants vs. healthy teeth }\end{array}$ & $\begin{array}{l}20 \text { patients ( } 10 \text { with healthy implants; } 10 \text { with at least } \\
1 \text { healthy implant, } 1 \text { peri-implantitis and periodontally } \\
\text { healthy teeth) }\end{array}$ & NS & $\begin{array}{l}\text { Higher Actinomyces, Atopobium, Gemella, Kingella and Rothia and lower Campylobacter, Desulfobulbus, Dialister, } \\
\text { Eubacterium, Filifactor, Mitsukella, Porphyromonas and Pseudoramibacter in healthy implants. } \\
\text { Higher } F \text {. nucleatum, Dialister invisus, Streptococcus sp. human oral taxon (HOT) 064, Fillifactor alocis and } \\
\text { Mitsuokella a } p \text {. HOT } 131 \text { and lower Veillonella dispar, Actinomyces meyeri, Granulicatella adiacens in peri- } \\
\text { implantitis. }\end{array}$ \\
\hline Maruyama et al. [70] & 2014 & $\begin{array}{l}\text { Peri-implant disease vs. } \\
\text { periodontal disease }\end{array}$ & 20 patients & 1 implant per patient & $\begin{array}{l}\text { Higher Olsenella, Sphingomonas, Peptostreptococcus, and unclassified Neisseriaceae and lower Desulfomicrobium } \\
\text { in peri-implantitis vs. periodontitis. } \\
\text { P. gingivalis, T. denticola, and T. forsythia were abundant and prevalent in both diseases. }\end{array}$ \\
\hline Kumar et al. [71] & 2012 & $\begin{array}{l}\text { Peri-implant health and } \\
\text { disease vs. periodontal } \\
\text { health and disease }\end{array}$ & $\begin{array}{l}40 \text { patients ( } 10 \text { peri-implantitis; } 10 \text { peri-implant health; } \\
10 \text { chronic periodontitis; } 10 \text { periodontal health) }\end{array}$ & 1 per patient & Significantly lower diversity of peri-implant biofilms than subgingival biofilms in both health and disease. \\
\hline Heuer et al. [72] & 2012 & $\begin{array}{l}\text { Peri-implant mucositis vs. } \\
\text { periodontal gingivitis }\end{array}$ & 9 patients & 1 per patient and condition & Higher diversity in gingival than peri-implant sulci. \\
\hline Dabdoub et al. [73] & 2013 & $\begin{array}{l}\text { Peri-implant health and } \\
\text { disease vs. periodontal } \\
\text { health and disease }\end{array}$ & $\begin{array}{l}81 \text { subjects ( } 33 \text { healthy tooth/healthy implant; } 23 \text { healthy } \\
\text { tooth/diseased implant; } 8 \text { diseased tooth/healthy implant; } \\
\qquad 17 \text { diseased tooth/diseased implant) }\end{array}$ & $\begin{array}{l}162 \text { sites ( } 56 \text { healthy teeth; } 13 \text { gingivitis; } \\
12 \text { periodontitis; } 41 \text { peri-implant health; } \\
20 \text { peri-implant mucositis; } 20 \text { peri-implantitis) }\end{array}$ & $\begin{array}{l}\text { Sixty percent of individuals share }<50 \% \text { of all species between their periodontal and peri-implant biofilms; } 85 \% \\
\text { of individuals share }<8 \% \text {. Distinct bacterial lineages associated with health and disease in teeth and implants. The } \\
\text { periodontal microbiome demonstrated significantly higher diversity than the implant. Staphylococcus and Treponema } \\
\text { were significantly associated with diseased implants, but not teeth. }\end{array}$ \\
\hline Zhang et al. [74] & 2015 & $\begin{array}{l}\text { Peri-implant disease vs. } \\
\text { periodontal disease }\end{array}$ & 20 patients (10 healthy; 10 chronic periodontitis) & 1 per patient & $\begin{array}{l}\text { Lower A.a., P. gingivalis, T. forsythia and T. denticola and higher Pseudomonas in peri-implant sites vs. chronic } \\
\text { periodontitis. Different SR1 genus, Brevundimonas, Catonella, Desulfovibrio, Mogibacterium, Peptostreptococcus, } \\
\text { Propionibacterium and Pseudomonas between the two groups. Higher bacterial diversity in teeth vs. implants. }\end{array}$ \\
\hline Schaumann et al. [88] & 2014 & $\begin{array}{l}\text { Peri-implant disease vs. } \\
\text { periodontal disease }\end{array}$ & $\begin{array}{l}7 \text { patients with bleeding periodontal and peri-implant } \\
\text { tissues and bone loss }\end{array}$ & 1 per patient and condition & $\begin{array}{l}\text { Streptococcacea, Rothia and Porphyromonas were the most abundant taxa in supramucosal or supragingival plaques } \\
\text { on implants and teeth. In submucosal plaques at implants, the most abundant taxa were Rothia, Streptococcaceae and } \\
\text { Porphyromonas. The most abundant subgingival bacteria on teeth were Prevotella, Streptococcaceae and TG5. }\end{array}$ \\
\hline Faveri et al. [89] & 2011 & $\begin{array}{l}\text { Peri-implantitis vs. healthy } \\
\text { implants vs. healthy teeth }\end{array}$ & $\begin{array}{l}50 \text { patients }(25 \text { healthy; } 25 \text { with at least } 1 \text { healthy implant, } \\
1 \text { peri-implantitis and periodontally healthy teeth) }\end{array}$ & NS & $\begin{array}{l}\text { Higher positive sites for Archaea in peri-implantitis than healthy implants or healthy teeth. No significant differences } \\
\text { in healthy teeth vs. healthy implants. }\end{array}$ \\
\hline Heuer et al. [90] & 2013 & $\begin{array}{l}\text { Peri-implant tissue at } \\
\text { implant-supported bar } \\
\text { attachments vs. implant- } \\
\text { fixed telescopic double } \\
\text { crown attachments vs. teeth }\end{array}$ & $\begin{array}{l}16 \text { patients ( } 8 \text { healthy implant-supported bar-attached } \\
\text { supraconstruction; } 8 \text { healthy implant-annhored telescopic } \\
\text { double crown attachments) }\end{array}$ & 1 implant per patient & No statistically significant differences. \\
\hline
\end{tabular}

NS $=$ not specified. 
Table 5. Studies using other techniques or a variety of them

\begin{tabular}{|c|c|c|c|c|c|c|}
\hline Study & $\begin{array}{c}\text { Year of } \\
\text { publication }\end{array}$ & $\begin{array}{c}\text { Type of } \\
\text { patients/sites }\end{array}$ & Detection method & $\begin{array}{c}\text { Number of } \\
\text { patients }\end{array}$ & $\begin{array}{c}\text { Number of } \\
\text { implants/sites }\end{array}$ & Outcome \\
\hline Ata-Ali et al. [91] & 2011 & $\begin{array}{c}\text { Peri-implant mucositis vs. } \\
\text { healthy implants }\end{array}$ & $\begin{array}{c}\text { Hybridization with specific P32 } \\
\text { arrays directed against the sRNA } \\
\text { ribosomal subunit }\end{array}$ & 34 patients & 90 patients & $\begin{array}{l}\text { Significantly greater } P \text {. gingivalis, A.a., } T \text {. forsythia and } T \text {. } \\
\text { denticola in mucositis. }\end{array}$ \\
\hline Ata-Ali et al. [92] & 2015 & $\begin{array}{l}\text { Peri-implantitis vs. } \\
\text { healthy implants }\end{array}$ & $\begin{array}{c}\begin{array}{c}\text { Hybridization with specific P32 } \\
\text { arrays directed against the sRNA } \\
\text { ribosomal subunit }\end{array} \\
\end{array}$ & \begin{tabular}{|c|}
35 patients ( 22 healthy; \\
13 with peri-implantitis)
\end{tabular} & $\begin{array}{l}78 \text { implants ( } 54 \text { healthy; } \\
24 \text { with peri-implantitis) }\end{array}$ & $\begin{array}{l}\text { Significantly greater periodontal pathogens in peri- } \\
\text { implantitis. }\end{array}$ \\
\hline Ata-Ali et al. [93] & 2013 & $\begin{array}{c}\text { Peri-implant mucositis vs. } \\
\text { healthy implants }\end{array}$ & $\begin{array}{c}\text { Hybridization with specific P32 } \\
\begin{array}{c}\text { arrays directed against the sRNA } \\
\text { ribosomal subunit }\end{array}\end{array}$ & $\begin{array}{c}34 \text { patients (22 healthy; } \\
\begin{array}{c}12 \text { with peri-implant } \\
\text { mucositis })\end{array}\end{array}$ & $\begin{array}{l}77 \text { implants ( } 54 \text { healthy; } \\
23 \text { with peri-implant } \\
\text { mucositis) }\end{array}$ & $\begin{array}{l}\text { No differences in } T \text {. forsythia, P. gingivalis, and } T \text {. } \\
\text { denticola. }\end{array}$ \\
\hline $\begin{array}{l}\text { Albertini et al. } \\
\text { [94] }\end{array}$ & 2015 & $\begin{array}{l}\text { Peri-implantitis vs. } \\
\text { periodontitis }\end{array}$ & PCR and Culture & 33 patients & 48 implants +48 teeth & $\begin{array}{l}\text { No significant differences of } P \text {. gingivalis, } T \text {. forsythia, } \\
P \text {. intermedia, or } T \text {. denticola. S. aureus, } P \text {. aeruginosa } \\
\text { and C. albicans present in } 15 \% \text { of the patients. }\end{array}$ \\
\hline \multirow{3}{*}{$\begin{array}{l}\text { Charalampakis et } \\
\text { al. [95] }\end{array}$} & \multirow{3}{*}{2012} & \multirow{3}{*}{ Peri-implantitis } & Culture & NS & 139 implants & $\begin{array}{l}\text { P. intermedia/P. nigrescens: the most representative in } \\
\text { magnitude; } S \text {. epidermidis more prevalent than } S \text {. aureus. } \\
\text { A.a.: not identified in } 10 \text { of } 161 \text { cases. } \\
\text { Fungi and enterococci: seldom found. }\end{array}$ \\
\hline & & & $\begin{array}{l}\text { Checkerboard DNA-DNA } \\
\text { hybridization }\end{array}$ & NS & 120 implants & $\begin{array}{l}\text { T. forsythia: the most prevalent followed by } T \text {. denticola. } \\
\text { P. gingivalis: less prevalent than } P \text {. intermedia, } P \text {. } \\
\text { nigrescens and } P \text {. endodontalis. } \\
\text { S. noxia and } A . a . \text { the least representative. } \\
\end{array}$ \\
\hline & & & Both & NS & 22 implants & $\begin{array}{l}\text { Culture was unable to detect any of the targeted species in } \\
18.6 \% \text { of the cases, whereas checkerboard only in } 0.7 \% \text {. }\end{array}$ \\
\hline \multirow{3}{*}{$\begin{array}{l}\text { Charalampakis et } \\
\text { al. [96] }\end{array}$} & \multirow{3}{*}{2011} & \multirow{3}{*}{ Peri-implantitis } & Culture & \multirow[b]{2}{*}{274 patients pre-treatment } & \multirow[b]{2}{*}{ NS } & $\begin{array}{l}\text { Detection frequencies: P. intermedia/P. nigrescens } \\
(27.3 \%), \text { AGNB }(18.6 \%), \text { A.a. }(6 \%)\end{array}$ \\
\hline & & & $\begin{array}{l}\text { Checkerboard DNA-DNA } \\
\text { hybridization }\end{array}$ & & & $\begin{array}{l}\text { Detection frequencies: T. forsythia }(37.3 \%), \text { T. denticola } \\
(31 \%), \text { P. nigrescens }(28.9 \%) \text {, P. endodontalis }(28.6 \%), P \text {. } \\
\text { intermedia }(25.4 \%), \text { A.a. }(4.2 \%)\end{array}$ \\
\hline & & & Culture & 27 patients post-treatment & NS & $\begin{array}{l}\text { Detection frequencies: AGNB (25.9\%), P. gingivalis } \\
(25.9 \%), \text { P. intermedia/P. nigrescens }(22.2 \%), \\
\text { Enterococci }(7.4 \%), \text { A.a. }(4 \%), \text { S. aureus }(0 \%), S . \\
\text { epidermidis }(0 \%), \text { Fungi }(0 \%)\end{array}$ \\
\hline $\begin{array}{l}\text { Van Assche et al. } \\
\text { [97] }\end{array}$ & 2011 & $\begin{array}{l}\text { Peri-implant vs. } \\
\text { periodontal health }\end{array}$ & \begin{tabular}{|c|}
$\begin{array}{c}\text { Culture, qPCR and checkerboard } \\
\text { DNA-DNA hybridization analysis } \\
\text { of } 40 \text { species }\end{array}$ \\
\end{tabular} & 18 patients & $\begin{array}{l}66 \text { implants (34 rough } \\
\text { surface; } 32 \text { machined } \\
\text { surface) }\end{array}$ & $\begin{array}{l}\text { No statistically significant differences between the two } \\
\text { implant designs or level of bone loss. Similar subgingival } \\
\text { composition around implants and teeth. }\end{array}$ \\
\hline
\end{tabular}

$\mathrm{NS}=$ not specified 
In terms of differences between teeth and implants, inconsistent results have been reported. Although Ebadian and co-workers [61] found no significant differences between healthy implants and teeth, other studies have identified some particular species, such as $T$. forsythia, $P$. gingivalis, $P$. intermedia, Parvimonas micra, and T. denticola, in higher concentration around implants [60]. In the presence of periodontal disease, differences between periodontitis and peri-implantitis sites, specifically in the concentrations of $T$. forsythia, $P$. intermedia and $C$. rectus, are clear [61]. The difference between periodontal gingivitis and peri-implant mucositis, however, was not significant in an experimentally induced gingivitis - mucositis study by Salvi and coworkers [흐]

Finally, 14 studies have been identified that used the 16S rRNA gene sequencing technique (Table 4). When comparing peri-implant healthy sites with diseased, 10-fold higher mean colony-forming units have been identified in peri-implantitis sites compared to healthy implant sites, with periodontopathic bacteria not being the only periodontal pathogens active in peri-implantitis [64]. In this sense, the microbiome of mucositis can be described as intermediate in nature between healthy and periimplantitis sites. In fact, the pathogens found in periimplantitis sites can already be seen at a moderate abundance in mucositis sites [65-67]. In this transition from health to mucositis, loss of several healthassociated species occurs, which interestingly in nonsmokers, is based on an increase in diversity [67].

The comparison between implants and teeth in several studies agrees that peri-implantitis sites present higher diversity than periodontitis sites [68-70]. In contrast, other studies have found significantly lower diversity of peri-implant biofilms than subgingival biofilms in both health and disease [71-74]. In this sense, Dabdoub and co-workers [73] found that less $50 \%$ of all species were shared between periodontal and periimplant biofilms in the majority of the patients $(60 \%)$. These numbers are more evident as less than $8 \%$ of species are found in both teeth and implants in more than $85 \%$ of patients [73]. This is, in the vast majority of patients, microbes at each site are different; therefore, the ecosystem can also be referred to be different.

\section{Risk of bias within studies}

As a summary of the risk of bias within studies, we must highlight the fact that most of the studies included were observational cross-sectional studies. Most of them made a sound selection of the comparison group (if present) and used valid and reliable measures. However, in most cases when a comparison group was present it was difficult to identify the method of balancing the groups by important potential confounding variables that in some cases were not even taken into account, including age, gender, presence of periodontal disease, concomitant intake of antibiotics or anti-inflammatory drugs, type of implant-abutment connection, or last periodontal therapy, among others. Because of this, it can be said that most of the studies in this topic contain a moderate risk of bias.

\section{Risk of bias across studies}

There were several limitations present in the current review. The main difficulty in the topic under study is the difference in the microbial detection technique. In addition, the latter consensus in disease classification, diagnosis and reporting is not always followed in the included studies, although they do report the conditions properly. Also, few studies analyse simultaneously the microbiome and the host, which indicate limited information on this important interactions. Thus, the lack of comparable studies in terms of design, patient selection, defect and systemic conditions and microbial analysis make it difficult to draw solid conclusions.

\section{DISCUSSION}

Most of the studies on microbiology around implants and teeth use paper points to collect the samples. A recent study concluded that paper points used for sampling can contain contaminating bacterial DNA [75]. Therefore, the use of paper points as sampling tool for microbial profiling should be substituted by other methods such as sterile curettes.

Efforts are being made on generating a complete analysis of the human microbiome, the most important of them being the Human Microbiome Project (HMP). It should not be surprising that approximately $26 \%$ of all bacteria in the human body are located in the oral cavity [76]. It has been calculated that the microbiota on a human body outnumbers our somatic cells by 10 -fold. Therefore, understanding the human microbiome, its distribution and evolution is key in the understanding of the human physiology, as have been highlighted in several reviews [77].

The studies developed under the HMP project try to answer the question on what are the synergistic activities between humans and microbes. Other questions come from an ecological point of view 
such as how stable and resilient is the microbiota over the course of a day within one individual, and during the course of his or her lifespan, how similar they are between members of a family, a human community, and between communities living in different environments, etc. [77]. These studies were proposed to use the deep analysis tools that were developed under the Human Genome Project: random shotgun sequencing procedures, targeted large-insert clone sequencing, and assessments of intra- and interindividual variation by using high-density microarrays [78].

Much of the current understanding of microbiomes comes from culture-based approaches. However, as much as $20 \%$ to $60 \%$ of the human-associated microbiome has been estimated to be uncultivable [76]. This obviously results in an underestimation of the diversity of the human microbiome. Therefore, new technologies were needed. Then, genomic analysis by checkerboard or PCR was introduced. PCR as the first technique of a group identified as "molecular techniques", was introduced in the 80s. It uses an enzymatic replication process of the DNA that will be later observed in a gel or quantified after a number of replication cycles [3]. These techniques overcame the limitations of culturing techniques and also allowed great time-saving. They also offer great sensitivity. Then, the DNA-DNA hybridization ('checkerboard') technique was applied in periodontal studies by Socransky et al. [79]. It allows the evaluation of large amounts of plaque samples for multitude of species on a single support membrane by hybridizing the DNA samples against whole genomic DNA probes, detecting up to 78 species at once [3]. The use of DNA-DNA hybridization highly improved the studies performed before using traditional culture techniques. The vast majority of the studies on peri-implantitis, obviously, focused on the species identified before on periodontitis sites, as a high similarity was suspected at the time. However, the main disadvantage of the PCR and DNA-DNA hybridization methods is the need to preselect probes for the bacteria to be investigated [3]. Therefore, there is a certain bias as the observer selects which and which not should be looked for and does not allow finding 'unexpected' microbiota. Thus, studies are really difficult to be compared. In addition, the quality of the results depends highly on the quality of the probe and the hybridization conditions. Consequently, although useful for exploratory studies, these techniques should be discarded in the future.

New studies are sequencing genomic libraries made from DNA extracted directly from the sample without "looking for" a specific organism, a method called "metagenomics" [80]. The several limitations mentioned above for the previous techniques can be overcome by this new technology. It mainly refers to the gene sequencing of the $16 \mathrm{~S}$ rRNA gene. This technique is able to identify universal and conserved targets with important and distinctive phylogenetic information in complex microbial communities. Within the specific field of Dentistry, the analysis of the $16 \mathrm{~S}$ ribosomal gene sequence is probably the tool providing the more comprehensive examination of the taxonomically heterogeneous community associated with periodontal health and disease, as well as peri-implant diseases [무]. However, the technique presents limitations in identifying differences at the strain level, which may actually be the level that distinguishes between health and disease [3]. This can be overcome by shotgun sequencing of the whole genome. To date, no studies on peri-implant disease have been identified using this methodology.

\section{Summary of evidence}

It has been reported that the microbial profile in the peri-implant sulcus and adjacent periodontal pockets is specific for peri-implantitis and have high predictive value [53]. However, periodontal pathogens have been identified in healthy, peri-implant mucositis, and peri-implantitis sites. Thus, it could be argued that these microorganisms are not strictly associated with peri-implantitis $[49,81]$ and other species not analysed in some of those studies may be involved in the pathogenesis of peri-implant diseases [65]. Furthermore, in some cases, microorganisms not associated with periodontal disease have been a common finding at implants with peri-implantitis [62]. Therefore, conventional pathogens in periodontal disease may not be the only microorganisms active in peri-implantitis [64].

In any case, based on this review and according to others, the microbiota around implants is complex [4]. Nonetheless, in general, higher amounts of microorganisms have been found in sites diagnosed with peri-implantitis than in healthy sites. Despite the geographic proximity and some common microorganisms, the microbiota of periodontal tissues is different than the one found around implants $[\underline{64}, \underline{68}, \underline{70}, \underline{73}]$. Also, as properly discussed by Charalampakis and Belibasakis []ㅡ, with whom we agree, it may sound logical to think that if the microbiota at teeth and implants share a limited space they should be similar. However, the differences in topography and immunological characteristics of periodontal and peri-implant tissues must also drive to the logical conclusion that the biofilms associated with these surfaces have to be different. 


\section{Limitations}

Most of the studies published so far used techniques such as culture, PCR and checkerboard DNA-DNA hybridization that limit the possibility to detect species not considered in the analysis. Metagenomics techniques must be used in future studies and findings correlated with specific host characteristics (site, defect, region and systemic conditions and background). In most cases comparison groups were not balanced by important potential confounding variables, including age, gender, presence of periodontal disease, concomitant intake of antibiotics or anti-inflammatory drugs, type of implant-abutment connection, or last periodontal therapy, among others.

\section{CONCLUSIONS}

Microbial profile of peri-implant diseases usually includes classic periodontopathogens. However, correlation between studies is difficult, particularly because of the use of different detection methods.
New metagenomic techniques to avoid detection bias and careful balance and description of the included patients and implants should be promoted for future studies.

\section{ACKNOWLEDGMENTS AND DISCLOSURE STATEMENTS}

The authors of this manuscript were partially supported by Research Groups \#CTS-138 and \#CTS583 (Junta de Andalucía, Spain). MPM is supported by the Andalucía Talent Hub Program from the Andalusian Knowledge Agency (co-funded by the European Union's Seventh Framework Program, Marie Skłodowska-Curie actions (COFUND - Grant Agreement $n^{\circ}$ 291780) and the Ministry of Economy, Innovation, Science and Employment of the Junta de Andalucía). This research received no specific grant from any funding agency in the public, commercial, or not-for-profit sectors.

The authors declare no conflict of interest, either directly or indirectly, in any of the products listed in the manuscript.

\section{REFERENCES}

1. Derks J, Tomasi C. Peri-implant health and disease. A systematic review of current epidemiology. J Clin Periodontol. 2015 Apr;42:S158-71. [Medline: 25495683] [doi: 10.1111/jcpe.12334]

2. Padial-Molina M, Suarez F, Rios HF, Galindo-Moreno P, Wang H-L. Guidelines for the diagnosis and treatment of peri-implant diseases. Int J Periodontics Restorative Dent. 2014 Dec;34(6):e102-11. [Medline: 25411744] [doi: 10.11607/prd.1994]

3. Charalampakis G, Belibasakis GN. Microbiome of peri-implant infections: Lessons from conventional, molecular and metagenomic analyses. Virulence. 2015 Apr;6(3):183-7. [Medline: 25654499] [doi: 10.4161/21505594.2014.980661]

4. Renvert S, Quirynen M. Risk indicators for peri-implantitis. A narrative review. Clin Oral Implants Res. 2015 Sep;26 Suppl 11:15-44. [Medline: 26385619] [doi: 10.1111/clr.12636]

5. Jepsen S, Berglundh T, Genco R, Aass AM, Demirel K, Derks J, Figuero E, Giovannoli JL, Goldstein M, Lambert F, Ortiz-Vigon A, Polyzois I, Salvi GE, Schwarz F, Serino G, Tomasi C, Zitzmann NU. Primary prevention of periimplantitis: managing peri-implant mucositis. J Clin Periodontol. 2015 Apr;42 Suppl 16:S152-7. [Medline: 25626479] [doi: 10.1111/jcpe.12369]

6. Rehman A, Hu J, Ott SJ, Grössner-Schreiber B. Microbial community composition on modified dental implant surfaces: an in vivo study. Int J Oral Maxillofac Implants. 2012 Aug;27(4):811-9. [Medline: 22848882]

7. Dorkhan M, Svensäter G, Davies JR. Salivary pellicles on titanium and their effect on metabolic activity in Streptococcus oralis. BMC Oral Health. 2013;13:32. [Medline: 23866104] [PMC free article: 3726426] [doi: 10.1186/1472-6831-13-32]

8. Franková J, Pivodová V, Růžička F, Tománková K, Šafářová K, Vrbková J, Ulrichová J. Comparing biocompatibility of gingival fibroblasts and bacterial strains on a different modified titanium discs. J Biomed Mater Res A. 2013 Oct;101(10):2915-24. [Medline: 23529774] [doi: 10.1002/jbm.a.34598]

9. Dorkhan M, Hall J, Uvdal P, Sandell A, Svensäter G, Davies JR. Crystalline anatase-rich titanium can reduce adherence of oral streptococci. Biofouling. 2014;30(6):751-9. [Medline: 24881929] [doi: 10.1080/08927014.2014.922962]

10. Violant D, Galofré M, Nart J, Teles RP. In vitro evaluation of a multispecies oral biofilm on different implant surfaces. Biomed Mater Bristol Engl. 2014 Jun;9(3):035007. [Medline: 24770899] [doi: 10.1088/1748-6041/9/3/035007]

11. Cochis A, Azzimonti B, Della Valle C, Chiesa R, Arciola CR, Rimondini L. Biofilm formation on titanium implants counteracted by grafting gallium and silver ions. J Biomed Mater Res A. 2015 Mar;103(3):1176-87. [Medline: 25044610] [doi: $\underline{10.1002 / \mathrm{jbm} . \mathrm{a} .35270]}$ 
12. Sahm N, Becker J, Santel T, Schwarz F. Non-surgical treatment of peri-implantitis using an air-abrasive device or mechanical debridement and local application of chlorhexidine: a prospective, randomized, controlled clinical study. J Clin Periodontol. 2011 Sep;38(9):872-8. [Medline: 21770995] [doi: 10.1111/j.1600-051X.2011.01762.x]

13. Schmage P, Thielemann J, Nergiz I, Scorziello TM, Pfeiffer P. Effects of 10 cleaning instruments on four different implant surfaces. Int J Oral Maxillofac Implants. 2012 Apr;27(2):308-17. [Medline: 22442769]

14. Idlibi AN, Al-Marrawi F, Hannig M, Lehmann A, Rueppell A, Schindler A, Jentsch H, Rupf S. Destruction of oral biofilms formed in situ on machined titanium (Ti) surfaces by cold atmospheric plasma. Biofouling. 2013;29(4):369-79. [Medline: 23574038] [doi: 10.1080/08927014.2013.775255]

15. Marotti J, Tortamano P, Cai S, Ribeiro MS, Franco JEM, de Campos TT. Decontamination of dental implant surfaces by means of photodynamic therapy. Lasers Med Sci. 2013 Jan;28(1):303-9. [Medline: 22790655] [doi: 10.1007/s10103-012-1148-6]

16. Yoo EM, Uhm SH, Kwon JS, Choi HS, Choi EH, Kim KM, Kim KN. The Study on Inhibition of Planktonic Bacterial Growth by Non-Thermal Atmospheric Pressure Plasma Jet Treated Surfaces for Dental Application. J Biomed Nanotechnol. 2015 Feb;11(2):334-41. [Medline: 26349309] [doi: 10.1166/jbn.2015.2030]

17. Lan SF, Kehinde T, Zhang X, Khajotia S, Schmidtke DW, Starly B. Controlled release of metronidazole from composite poly- $\varepsilon$-caprolactone/alginate (PCL/alginate) rings for dental implants. Dent Mater. 2013 Jun;29(6):656-65. [Medline: 23602170] [doi: 10.1016/j.dental.2013.03.014]

18. He S, Zhou P, Wang L, Xiong X, Zhang Y, Deng Y, Wei S. Antibiotic-decorated titanium with enhanced antibacterial activity through adhesive polydopamine for dental/bone implant. J R Soc Interface. 2014 Mar 19;11(95):20140169. [Medline: 24647910] [PMC free article: 4006258] [doi: 10.1098/rsif.2014.0169]

19. Wood NJ, Jenkinson HF, Davis SA, Mann S, O’Sullivan DJ, Barbour ME. Chlorhexidine hexametaphosphate nanoparticles as a novel antimicrobial coating for dental implants. J Mater Sci Mater Med. 2015 Jun;26(6):201. [Medline: 26123234] [PMC free article: 4486094] [doi: 10.1007/s10856-015-5532-1]

20. Tripodi D, Vantaggiato G, Scarano A, Perrotti V, Piattelli A, Iezzi G, D’Ercole S. An in vitro investigation concerning the bacterial leakage at implants with internal hexagon and Morse taper implant-abutment connections. Implant Dent. 2012 Aug;21(4):335-9. [Medline: 22814560] [doi: 10.1097/ID.0b013e31825cd472]

21. Silva-Neto JP, Prudente MS, Carneiro Tde A, Nóbilo MA, Penatti MP, Neves FD. Micro-leakage at the implant-abutment interface with different tightening torques in vitro. J Appl Oral Sci. 2012 Sep-Oct;20(5):581-7. [Medline: 23138747] [PMC free article: 3881787] [doi: 10.1590/S1678-77572012000500015]

22. Pimentel AC, Manzi MR, Sartori SG, da Graça Naclério-Homem M, Sendyk WR. In vivo effectiveness of silicone gel sheets as barriers at the inner microgap between a prosthetic abutment and an external-hexagon implant platform. Int J Oral Maxillofac Implants. 2014 Feb;29(1):121-6. [Medline: 24451862] [doi: 10.11607/jomi.3177]

23. Smith NA, Turkyilmaz I. Evaluation of the sealing capability of implants to titanium and zirconia abutments against Porphyromonas gingivalis, Prevotella intermedia, and Fusobacterium nucleatum under different screw torque values. J Prosthet Dent. 2014 Sep;112(3):561-7. [Medline: 24656409] [doi: 10.1016/j.prosdent.2013.11.010]

24. Carcuac O, Derks J, Charalampakis G, Abrahamsson I, Wennström J, Berglundh T. Adjunctive Systemic and Local Antimicrobial Therapy in the Surgical Treatment of Peri-implantitis A Randomized Controlled Clinical Trial. J Dent Res. 2016 Jan 1;95(1):50-7. [Medline: 26285807] [doi: 10.1177/0022034515601961]

25. Renvert S, Polyzois I. Risk indicators for peri-implant mucositis: a systematic literature review. J Clin Periodontol. 2015;42:S172-86. [Medline: 25496066] [doi: 10.1111/jcpe.12346]

26. Koyanagi T, Sakamoto M, Takeuchi Y, Ohkuma M, Izumi Y. Analysis of microbiota associated with peri-implantitis using 16S rRNA gene clone library. J Oral Microbiol. 2010 May 24;2. [Medline: 21523229] [PMC free article: $\underline{3084566]}$ [doi: $10.3402 /$ jom.v2i0.5104]

27. Mombelli A, Décaillet F. The characteristics of biofilms in peri-implant disease: Biofilms in peri-implant disease. J Clin Periodontol. 2011 Mar;38:203-13. [Medline: 21323716] [doi: 10.1111/j.1600-051X.2010.01666.x]

28. Moher D, Liberati A, Tetzlaff J, Altman DG, Group TP. Preferred Reporting Items for Systematic Reviews and MetaAnalyses: The PRISMA Statement. PLOS Med. 2009 Jul 21;6(7):e1000097. [Medline: 19621072] [PMC free article: 2707599] [doi: 10.1371/journal.pmed.1000097]

29. Sanz M, Lang NP, Kinane DF, Berglundh T, Chapple I, Tonetti MS. Seventh European Workshop on Periodontology of the European Academy of Periodontology at the Parador at la Granja, Segovia, Spain. J Clin Periodontol. 2011 Mar;38 Suppl 11:1-2. [Medline: 21323697] [doi: 10.1111/j.1600-051X.2010.01692.x]

30. Viswanathan M, Berkman ND, Dryden DM, Hartling L. Assessing Risk of Bias and Confounding in Observational Studies of Interventions or Exposures: Further Development of the RTI Item Bank [Internet]. Rockville (MD): Agency for Healthcare Research and Quality (US); 2013 Aug. [Medline: 24006553] [URL: http://www.ncbi.nlm.nih.gov/books/NBK154461/]

31. Enkling N, Jöhren P, Klimberg V, Bayer S, Mericske-Stern R, Jepsen S. Effect of platform switching on peri-implant bone levels: a randomized clinical trial. Clin Oral Implants Res. 2011 Oct;22(10):1185-92. [Medline: 21320171] [doi: 10.1111/j.1600-0501.2010.02090.x] 
32. Harder S, Podschun R, Grancicova L, Mehl C, Kern M. Analysis of the intraimplant microflora of two-piece dental implants. Clin Oral Investig. 2013 May;17(4):1135-42. [Medline: 22961460] [doi: 10.1007/s00784-012-0805-2]

33. Mawhinney J, Connolly E, Claffey N, Moran G, Polyzois I. An in vivo comparison of internal bacterial colonization in two dental implant systems: identification of a pathogenic reservoir. Acta Odontol Scand. 2015 Apr;73(3):188-94. [Medline: 25385682] [doi: 10.3109/00016357.2014.978365]

34. Zhuang LF, Watt RM, Steiner S, Lang-Hua BH, Wang R, Ramseier CA, Lang NP. Subgingival microbiota of Sri Lankan tea labourers naïve to oral hygiene measures. J Clin Periodontol. 2014 May;41(5):433-41. [Medline: 24460707] [doi: 10.1111/jepe.12230]

35. Korsch M, Walther W, Marten SM, Obst U. Microbial analysis of biofilms on cement surfaces: An investigation in cement-associated peri-implantitis. J Appl Biomater Funct Mater. 2014 Sep 5;12(2):70-80. [Medline: 24980683] [doi: $10.5301 /$ jabfm.5000206]

36. Manni A, Spalletta E, Arena M, Cattani P, Marchetta S, Celletti R. Microbiologic evaluation of crevicular fluid in patients treated with platform switching and traditional implants. J Biol Regul Homeost Agents. 2012 Jun;26(2 Suppl):9-17. [Medline: 23164322]

37. Canullo L, Tallarico M, Radovanovic S, Delibasic B, Covani U, Rakic M. Distinguishing predictive profiles for patientbased risk assessment and diagnostics of plaque induced, surgically and prosthetically triggered peri-implantitis. Clin Oral Implants Res. 2015 Nov 20. [Medline: 26584716] [doi: 10.1111/clr.12738]

38. Mussano F, Rovasio S, Schierano G, Baldi I, Carossa S. The effect of glycine-powder airflow and hand instrumentation on peri-implant soft tissues: a split-mouth pilot study. Int J Prosthodont. 2013 Feb;26(1):42-4. [doi: 10.11607/ijp.3063] [Medline: 23342332]

39. Swierkot K, Lottholz P, Flores-de-Jacoby L, Mengel R. Mucositis, Peri-Implantitis, Implant Success, and Survival of Implants in Patients With Treated Generalized Aggressive Periodontitis: 3- to 16-Year Results of a Prospective LongTerm Cohort Study. J Periodontol. 2012 Jan;83(10):1213-25. [Medline: 22264211] [doi: 10.1902/jop.2012.110603]

40. Ahmed A, Chambers MS, Goldschmidt MC, Habib A, Lei X, Jacob RF. Association between microbial flora and tissue abnormality around dental implants penetrating the skin in reconstructed oral cancer patients. Int J Oral Maxillofac Implants. 2012 Jun;27(3):684-94. [Medline: 22616064]

41. de Freitas AOA, Alviano CS, Alviano DS, Siqueira JF, Nojima LI, Nojima M da CG. Microbial colonization in orthodontic mini-implants. Braz Dent J. 2012;23(4):422-7. [Medline: 23207860] [doi: 10.1590/S0103-64402012000400019]

42. Obst U, Marten S-M, Niessner C, Hartwig E, Korsch M, Walther W. Diversity of patients microflora on orthopaedic and dental implants. Int J Artif Organs. 2012 Oct;35(10):727-34. [Medline: 23138700]

43. Tortamano A, Dominguez GC, Haddad ACSS, Nunes FD, Nacao M, Morea C. Periodontopathogens around the surface of mini-implants removed from orthodontic patients. Angle Orthod. 2012 Jul;82(4):591-5. [Medline: 22839769] [doi: $10.2319 / 081011-506.1]$

44. Rams TE, Balkin BE, Roberts TW, Molzan AK. Microbiological aspects of human mandibular subperiosteal dental implants. J Oral Implantol. 2013 Dec;39(6):714-22. [Medline: 21767207] [doi: 10.1563/AAID-JOI-D-11-00023]

45. Asadzadeh N, Naderynasab M, Fard FG, Rohi A, Haghi HR. The microbiology of the peri-implant sulcus following successful implantation of oral prosthetic treatments. Indian J Dent Res. 2012 Dec;23(6):753-7. [Medline: 23649058] [doi: 10.4103/0970-9290.111253]

46. Neilands J, Wickström C, Kinnby B, Davies JR, Hall J, Friberg B, Svensäter G. Bacterial profiles and proteolytic activity in peri-implantitis versus healthy sites. Anaerobe. 2015 Oct;35(Pt A):28-34. doi:. [Medline: 25870134] [doi: 10.1016/j.anaerobe.2015.04.004]

47. Tripodakis A-P, Nakou M. Microbiologic evaluation of compromised periodontal sites before and after immediate intrasocket implant placement. Int J Periodontics Restorative Dent. 2011 Dec;31(6):e109-17. [Medline: 22140671]

48. de Waal YCM, Raghoebar GM, Huddleston Slater JJR, Meijer HJA, Winkel EG, van Winkelhoff AJ. Implant decontamination during surgical peri-implantitis treatment: a randomized, double-blind, placebo-controlled trial. J Clin Periodontol. 2013 Feb;40(2):186-95. [Medline: 23211012] [doi: 10.1111/jcpe.12034]

49. Casado PL, Otazu IB, Balduino A, de Mello W, Barboza EP, Duarte MEL. Identification of periodontal pathogens in healthy periimplant sites. Implant Dent. 2011 Jun;20(3):226-35. [Medline: 21613949] [doi: 10.1097/ID.0b013e3182199348]

50. Canullo L, Pe-arrocha-Oltra D, Covani U, Botticelli D, Serino G, Penarrocha M. Clinical and microbiological findings in patients with peri-implantitis: a cross-sectional study. Clin Oral Implants Res. 2016 Mar;27(3):376-82. [Medline: 25622536] [doi: 10.1111/clr.12557]

51. Wang HL, Garaicoa-Pazmino C, Collins A, Ong HS, Chudri R, Giannobile WV. Protein biomarkers and microbial profiles in peri-implantitis. Clin Oral Implants Res. 2016 Sep;27(9):1129-36. [Medline: 26424287] [doi: 10.1111/clr.12708]

52. Canullo L, Pe-arrocha-Oltra D, Covani U, Rossetti PHO. Microbiologic and clinical findings of implants in healthy condition and with peri-implantitis. Int J Oral Maxillofac Implants. 2015 Aug;30(4):834-42. [Medline: 26252036] [doi: 10.11607/jomi.3947]

53. Canullo L, Radovanović S, Delibasic B, Blaya JA, Penarrocha D, Rakic M. The predictive value of microbiological findings on teeth, internal and external implant portions in clinical decision making. Clin Oral Implants Res. 2016 Apr 14. [Medline: 27079924] [doi: 10.1111/clr.12828] 
54. Zhuang L-F, Watt RM, Mattheos N, Si M-S, Lai H-C, Lang NP. Periodontal and peri-implant microbiota in patients with healthy and inflamed periodontal and peri-implant tissues. Clin Oral Implants Res. 2016 Jan;27(1):13-21. [Medline: 25399962] [doi: 10.1111/clr.12508]

55. Aoki M, Takanashi K, Matsukubo T, Yajima Y, Okuda K, Sato T, Ishihara K. Transmission of periodontopathic bacteria from natural teeth to implants. Clin Implant Dent Relat Res. 2012 Jun;14(3):406-11. [Medline: 20002682] [doi: $10.1111 / \mathrm{j} .1708-8208.2009 .00260 . \mathrm{x}]$

56. Cosgarea R, Dannewitz B, Sculean A, Bran S, Rotaru H, Baciut G, Eick S. Bacterial and inflammatory behavior of implants in the early healing phase of chronic periodontitis. Quintessence Int. 2012 Jun;43(6):491-501. [Medline: 22532956]

57. Sato J, Gomi K, Makino T, Kawasaki F, Yashima A, Ozawa T, Maeda N, Arai T. The evaluation of bacterial flora in progress of peri-implant disease. Aust Dent J. 2011 Jun;56(2):201-6. [Medline: 21623813] [doi: $10.1111 / \mathrm{j} .1834-7819.2011 .01324 . x$ ]

58. Cosyn J, Van Aelst L, Collaert B, Persson GR, De Bruyn H. The peri-implant sulcus compared with internal implant and suprastructure components: a microbiological analysis. Clin Implant Dent Relat Res. 2011 Dec;13(4):286-95. [Medline: 19673920] [doi: 10.1111/j.1708-8208.2009.00220.x]

59. De Bruyn H, Bouvry P, Collaert B, De Clercq C, Persson GR, Cosyn J. Long-term clinical, microbiological, and radiographic outcomes of Brånemark ${ }^{\mathrm{TM}}$ implants installed in augmented maxillary bone for fixed full-arch rehabilitation. Clin Implant Dent Relat Res. 2013 Feb;15(1):73-82. [Medline: 21745320] [doi: 10.1111/j.1708-8208.2011.00359.x]

60. Dierens M, Vandeweghe S, Kisch J, Persson GR, Cosyn J, De Bruyn H. Long-term follow-up of turned single implants placed in periodontally healthy patients after 16 to 22 years: microbiologic outcome. J Periodontol. 2013 Jul;84(7): 880-94. [Medline: 22934839] [doi: 10.1902/jop.2012.120187]

61. Ebadian AR, Kadkhodazadeh M, Zarnegarnia P, Dahlén G. Bacterial analysis of peri-implantitis and chronic periodontitis in Iranian subjects. Acta Med Iran. 2012;50(7):486-92. [Medline: 22930382]

62. Persson GR, Renvert S. Cluster of bacteria associated with peri-implantitis. Clin Implant Dent Relat Res. 2014 Dec;16(6):783-93. [Medline: 23527870] [doi: 10.1111/cid.12052]

63. Salvi GE, Aglietta M, Eick S, Sculean A, Lang NP, Ramseier CA. Reversibility of experimental peri-implant mucositis compared with experimental gingivitis in humans. Clin Oral Implants Res. 2012 Feb;23(2):182-90. [Medline: 21806683] [doi: 10.1111/j.1600-0501.2011.02220.x]

64. Tamura N, Ochi M, Miyakawa H, Nakazawa F. Analysis of bacterial flora associated with peri-implantitis using obligate anaerobic culture technique and 16S rDNA gene sequence. Int J Oral Maxillofac Implants. 2013 Dec;28(6):1521-9. [Medline: 24278920] [doi: 10.11607/jomi.2570]

65. Cortelli SC, Cortelli JR, Romeiro RL, Costa FO, Aquino DR, Orzechowski PR, Araújo VC, Duarte PM. Frequency of periodontal pathogens in equivalent peri-implant and periodontal clinical statuses. Arch Oral Biol. 2013 Jan;58(1):67-74. [Medline: 23127822] [doi: 10.1016/j.archoralbio.2012.09.004]

66. Zheng H, Xu L, Wang Z, Li L, Zhang J, Zhang Q, Chen T, Lin J, Chen F. Subgingival microbiome in patients with healthy and ailing dental implants. Sci Rep. 2015 Jun 16;5:10948. [Medline: 26077225] [PMC free article: 4468443] [doi: $10.1038 /$ srep 10948]

67. Tsigarida AA, Dabdoub SM, Nagaraja HN, Kumar PS. The influence of smoking on the peri-implant microbiome. J Dent Res. 2015 Sep;94(9):1202-17. [Medline: 26124222] [doi: 10.1177/0022034515590581]

68. Koyanagi T, Sakamoto M, Takeuchi Y, Maruyama N, Ohkuma M, Izumi Y. Comprehensive microbiological findings in peri-implantitis and periodontitis. J Clin Periodontol. 2013 Mar 1;40(3):218-26. [Medline: 23294017] [doi: $10.1111 /$ jepe.12047]

69. da Silva ESC, Feres M, Figueiredo LC, Shibli JA, Ramiro FS, Faveri M. Microbiological diversity of periimplantitis biofilm by Sanger sequencing. Clin Oral Implants Res. 2014 Oct;25(10):1192-9. [Medline: 23845046] [doi: $10.1111 / \mathrm{clr} .12231]$

70. Maruyama N, Maruyama F, Takeuchi Y, Aikawa C, Izumi Y, Nakagawa I. Intraindividual variation in core microbiota in peri-implantitis and periodontitis. Sci Rep. 2014 Oct 13;4:6602. [Medline: 25308100] [PMC free article: 4194447] [doi: 10.1038/srep06602]

71. Kumar PS, Mason MR, Brooker MR, O’Brien K. Pyrosequencing reveals unique microbial signatures associated with healthy and failing dental implants. J Clin Periodontol. 2012 May;39(5):425-33. [Medline: 22417294] [PMC free article: 3323747] [doi: 10.1111/j.1600-051X.2012.01856.x]

72. Heuer W, Kettenring A, Stumpp SN, Eberhard J, Gellermann E, Winkel A, Stiesch M. Metagenomic analysis of the peri-implant and periodontal microflora in patients with clinical signs of gingivitis or mucositis. Clin Oral Investig. 2012 Jun;16(3):843-50. [Medline: 21538072] [doi: 10.1007/s00784-011-0561-8]

73. Dabdoub SM, Tsigarida AA, Kumar PS. Patient-specific analysis of periodontal and peri-implant microbiomes. J Dent Res. 2013 Dec;92(12 Suppl):168S - 75S. [Medline: 24158341] [PMC free article: 3827621] [doi: 10.1177/0022034513504950]

74. Zhang Q, Qin XY, Jiang WP, Zheng H, Xu XL, Chen F. Comparison of Subgingival and Peri-implant Microbiome in Chronic Periodontitis. Chin J Dent Res. 2015 Sep;18(3):155-62. [Medline: 26485507]

75. van der Horst J, Buijs MJ, Laine ML, Wismeijer D, Loos BG, Crielaard W, Zaura E. Sterile paper points as a bacterial DNA-contamination source in microbiome profiles of clinical samples. J Dent. 2013 Dec;41(12):1297-301. [Medline: 24135296] [doi: 10.1016/j.jdent.2013.10.008] 
76. NIH HMP Working Group, Peterson J, Garges S, Giovanni M, McInnes P, Wang L, Schloss JA, Bonazzi V, McEwen JE, Wetterstrand KA, Deal C, Baker CC, Di Francesco V, Howcroft TK, Karp RW, Lunsford RD, Wellington CR, Belachew T, Wright M, Giblin C, David H, Mills M, Salomon R, Mullins C, Akolkar B, Begg L, Davis C, Grandison L, Humble M, Khalsa J, Little AR, Peavy H, Pontzer C, Portnoy M, Sayre MH, Starke-Reed P, Zakhari S, Read J, Watson B, Guyer M. The NIH Human Microbiome Project. Genome Res. 2009 Dec;19(12):2317-23. [Medline: 19819907] [PMC free article: 2792171] [doi: 10.1101/gr.096651.109]

77. Turnbaugh PJ, Ley RE, Hamady M, Fraser-Liggett C, Knight R, Gordon JI. The human microbiome project: exploring the microbial part of ourselves in a changing world. Nature. 2007 Oct 18;449(7164):804-10. [Medline: 17943116] [PMC free article: $\underline{3709439}$ ] [doi: 10.1038/nature06244]

78. Relman DA. New Technologies, Human-Microbe Interactions, and the Search for Previously Unrecognized Pathogens. J Infect Dis. 2002 Dec 1;186(Supplement 2):S254-8. [Medline: 12424706] [doi: 10.1086/344935]

79. Socransky SS, Smith C, Martin L, Paster BJ, Dewhirst FE, Levin AE. "Checkerboard" DNA-DNA hybridization. BioTechniques. 1994 Oct;17(4):788-92. [Medline: 7833043]

80. Handelsman J, Rondon MR, Brady SF, Clardy J, Goodman RM. Molecular biological access to the chemistry of unknown soil microbes: a new frontier for natural products. Chem Biol. 1998 Oct;5(10):R245-9. [Medline: 9818143] [doi: 10.1016/S1074-5521(98)90108-9]

81. Casado PL, Pereira MC, Duarte ME, Granjeiro JM. History of chronic periodontitis is a high risk indicator for periimplant disease. Braz Dent J. 2013;24(2):136-41. [Medline: 23780361] [doi: 10.1590/0103-6440201302006]

82. Canullo L, Rossetti PHO, Penarrocha D. Identification of Enterococcus faecalis and Pseudomonas aeruginosa on and in implants in individuals with peri-implant disease: a cross-sectional study. Int J Oral Maxillofac Implants. 2015 Jun;30(3):583-7. [Medline: 26009909] [doi: 10.11607/jomi.3946]

83. Jankovic S, Aleksic Z, Dimitrijevic B, Lekovic V, Milinkovic I, Kenney B. Correlation between different genotypes of human cytomegalovirus and Epstein-Barr virus and peri-implant tissue status. Aust Dent J. 2011 Dec;56(4):382-8. [Medline: 22126347] [doi: 10.1111/j.1834-7819.2011.01360.x]

84. Swierkot K, Brusius M, Leismann D, Nonnenmacher C, Nüsing R, Lubbe D, Schade-Brittinger C, Mengel R. Manual versus sonic-powered toothbrushing for plaque reduction in patients with dental implants: an explanatory randomised controlled trial. Eur J Oral Implantol. 2013 Summer;6(2):133-44. [Medline: 23926585]

85. van Brakel R, Cune MS, van Winkelhoff AJ, de Putter C, Verhoeven JW, van der Reijden W. Early bacterial colonization and soft tissue health around zirconia and titanium abutments: an in vivo study in man. Clin Oral Implants Res. 2011 Jun;22(6):571-7. [Medline: 21054554] [doi: 10.1111/j.1600-0501.2010.02005.x]

86. Hallström H, Persson GR, Lindgren S, Olofsson M, Renvert S. Systemic antibiotics and debridement of periimplant mucositis. A randomized clinical trial. J Clin Periodontol. 2012 Jun;39(6):574-81. [Medline: 22571225] [doi: 10.1111/j.1600-051X.2012.01884.x]

87. Tsoukaki M, Kalpidis CDR, Sakellari D, Tsalikis L, Mikrogiorgis G, Konstantinidis A. Clinical, radiographic, microbiological, and immunological outcomes of flapped vs. flapless dental implants: a prospective randomized controlled clinical trial. Clin Oral Implants Res. 2013 Sep;24(9):969-76. [Medline: 22708917]

88. Schaumann S, Staufenbiel I, Scherer R, Schilhabel M, Winkel A, Stumpp SN, Eberhard J, Stiesch M. Pyrosequencing of supra- and subgingival biofilms from inflamed peri-implant and periodontal sites. BMC Oral Health. 2014 Dec 17;14:157. [Medline: 25518856] [PMC free article: 4298060] [doi: 10.1186/1472-6831-14-157]

89. Faveri M, Gonçalves LF, Feres M, Figueiredo LC, Gouveia LA, Shibli JA, Mayer MP. Prevalence and microbiological diversity of Archaea in peri-implantitis subjects by 16S ribosomal RNA clonal analysis. J Periodontal Res. 2011 Jun;46(3):338-44. [Medline: 21338359] [doi: 10.1111/j.1600-0765.2011.01347.x]

90. Heuer W, Kettenring A, Demling A, Stumpp SN, Gellermann E, Winkel A, Stiesch M. Microbial diversity of peri-implant biofilms on implant fixed bar and telescopic double crown attachments. J Oral Implantol. 2013 Dec;39(6):648-54. [Medline: 21332328] [doi: 10.1563/AAID-JOI-D-10-00088]

91. Ata-Ali J, Flichy-Fernández AJ, Alegre-Domingo T, Candel-Marti ME, Peñarrocha D, Balaguer-Martinez JF, Peñarrocha MA. Analysis of the peri-implant microbiota in 90 dental implants and its relationship to crevicular fluid volume. Med Oral Patol Oral Cir Bucal. 2011 Nov 1;16(7):e944-7. [Medline: 21743411] [doi: 10.4317/medoral.17231]

92. Ata-Ali J, Flichy-Fernández AJ, Alegre-Domingo T, Ata-Ali F, Palacio J, Pe-arrocha-Diago M. Clinical, microbiological, and immunological aspects of healthy versus peri-implantitis tissue in full arch reconstruction patients: a prospective cross-sectional study. BMC Oral Health. 2015;15:43. [Medline: 25888355] [PMC free article: 4391105] [doi: $10.1186 /$ s12903-015-0031-9]

93. Ata-Ali J, Flichy-Fernandez AJ, Ata-Ali F, Penarrocha-Diago M, Penarrocha-Diago M. Clinical, microbiologic, and host response characteristics in patients with peri-implant mucositis. Int J Oral Maxillofac Implants. 2013 Jun;28(3):883-90. [Medline: 23748323] [doi: 10.11607/jomi.2654]

94. Albertini M, López-Cerero L, O’Sullivan MG, Chereguini CF, Ballesta S, Ríos V, Herrero-Climent M, Bullón P. Assessment of periodontal and opportunistic flora in patients with peri-implantitis. Clin Oral Implants Res. 2015 Aug;26(8):937-41. [Medline: 24720498] [doi: 10.1111/clr.12387] 
95. Charalampakis G, Leonhardt Å, Rabe P, Dahlén G. Clinical and microbiological characteristics of peri-implantitis cases: a retrospective multicentre study. Clin Oral Implants Res. 2012 Sep;23(9):1045-54. [Medline: 22092339] [doi: $10.1111 / \mathrm{j} .1600-0501.2011 .02258 . \mathrm{x}$ ]

96. Charalampakis G, Rabe P, Leonhardt A, Dahlén G. A follow-up study of peri-implantitis cases after treatment. J Clin Periodontol. 2011 Sep;38(9):864-71. [Medline: 21770994] [doi: 10.1111/j.1600-051X.2011.01759.x]

97. Van Assche N, Pittayapat P, Jacobs R, Pauwels M, Teughels W, Quirynen M. Microbiological outcome of two screwshaped titanium implant systems placed following a split-mouth randomised protocol, at the 12th year of follow-up after loading. Eur J Oral Implantol. 2011;4(2):103-16. [Medline: 21808760]

\section{To cite this article:}

Padial-Molina M, López-Martínez J, O’Valle F, Galindo-Moreno P.

Microbial Profiles and Detection Techniques in Peri-Implant Diseases: a Systematic Review

J Oral Maxillofac Res 2016;7(3):e10

URL: http://www.ejomr.org/JOMR/archives/2016/3/e10/v7n3e10.pdf

doi: $10.5037 /$ jomr.2016.7310

Copyright (C) Padial-Molina M, López-Martínez J, O’Valle F, Galindo-Moreno P. Published in the JOURNAL OF ORAL \& MAXILLOFACIAL RESEARCH (http://www.ejomr.org), 9 September 2016.

This is an open-access article, first published in the JOURNAL OF ORAL \& MAXILLOFACIAL RESEARCH, distributed under the terms of the Creative Commons Attribution-Noncommercial-No Derivative Works 3.0 Unported License, which permits unrestricted non-commercial use, distribution, and reproduction in any medium, provided the original work and is properly cited. The copyright, license information and link to the original publication on (http://www.ejomr.org) must be included. 